\title{
Matching Web Site Structure and Content
}

\author{
Vassil Gedov ${ }^{1}$ \\ Carsten Stolz ${ }^{2}$ \\ Ralph Neuneier ${ }^{3}$ \\ Michal Skubacz ${ }^{3}$ \\ Dietmar Seipel ${ }^{1}$ \\ \{gedov,seipel\}@informatik.uni-wuerzburg.de, University of Würzburg, Germany1 \\ carsten.stolz@ku-eichstaett.de, University of Eichstätt-Ingolstadt, Germany² \\ \{ralph.neuneier,michal.skubacz\}@siemens.com, Siemens AG, Corporate Technology, Germany ${ }^{3}$
}

\begin{abstract}
To keep an overview of a complex corporate web sites, it is crucial to understand the relationship of contents, structure and the user's behavior. In this paper, we describe an approach which is allowing us to compare web page content with the information implictly defined by the structure of the web site. We start by describing each web page with a set of key words. We combine this information with the link structure in an algorithm generating a context based description. By comparing both descriptions, we draw conclusions about the semantic relationship of a web page and its neighbourhood. In this way, we indicate whether a page fits in the content of its neighbourhood. Doing this, we implicitly identify topics which span over several connected web pages. With our approach we support redesign processes by assessing the actual structure and content of a web site with designer's concepts.
\end{abstract}

\section{Categories and Subject Descriptors}

H.3.3 [Information Systems]: Information search and retrieval; I.5.4 [Computing methodologies]: Applications

\section{General Terms}

Algorithms

\section{Keywords}

Web Structure, Web Content Mining, Semantic Description

\section{INTRODUCTION}

Facing the complexity of dynamically generated corporate web sites, it becomes increasingly difficult to understand user navigation patterns without deep knowledge of the content and the semantical linkage of the pages. Another challenge for web site owners as well as web strategists is to keep track of the information structures on the web site in the content management system. Following recent research concerning integration of web structure, content and

Permission to make digital or hard copies of all or part of this work for personal or classroom use is granted without fee provided that copies are not made or distributed for profit or commercial advantage and that copies bear this notice and the full citation on the first page. To copy otherwise, to republish, to post on servers or to redistribute to lists, requires prior specific permission and/or a fee.

Copyright 200X ACM X-XXXXX-XX-X/XX/XX ...\$5.00. usage mining [1] [2], we intend to provide an insight into the relationship between the structure and the content. We first try to extract the key content from each page taking its hypertext markup into account. Then, we reason about the role of the web structure[3] since we believe that the linkage created by the web designer conceals a semantic connection between web pages.

\section{COMBINING STRUCTURE AND CON- TENT INFORMATION}

Before associating structure and content with each other, we will try to define them.

The structure of a web site is determined by the link structure between its web pages. A link in this context is considered to be a link encoded as a HTML tag. We do not consider the anchor text of a link as structural information since we believe it belongs to the content information. Excluding self-references, we focus on hard coded inter-page links represented by a directed cyclic graph.

The content of a web site may consist of text, hypertext, meta information or multimedia content like pictures, figures, video or sound. Here, we concentrate on text and html since they contain most of the information. As more and more web sites use different media types it could be reasonable to include those in future work .

In order to combine structure and content, we have to map structural and content information to a common concept. We achieve it by putting them in a common data structure, in order to make them comparable. Next, we combine the textual content of a web page with linked contents, and thus we implicitly create a contentstructure graph.

\section{ALGORITHM}

In this section we describe how to create a content-structure graph and compare it with the structure graph of a web site. Thereafter, we present a way to measure the distance between both.

\subsection{Structure and Content Description}

From each page we extract the links within a web site generating an implicit directed cyclic structure graph. We consider siteinternal links for each web site, respectively. In order to describe the content, we extract all words and stem them. Further more, stop words are filtered as we are only interested in content, and not in style or grammatical information. Next, we calculate word frequencies per web page. We assume that the most frequent words define the content of the web page. The highest ranked words are said to be key words, where their number is proportional to the text length. Not all key words contribute to the semantic: very frequent key 
